\title{
Did Undergraduate Students Really Establish Reversible Reasoning When Faced With Inverse Function Problem Situations?
}

\author{
Muhammad Ikram ${ }^{*}$, Hery Susanto ${ }^{2}$, Purwanto $^{3}$, I Nengah Parta ${ }^{4}$ \\ ${ }^{1}$ Department of Mathematics Education, Universitas Cokroaminoto Palopo, South Sulawesi, Indonesia \\ E-mail: muhammad.ikram.1603119@students.um.ac.id \\ ${ }^{2,3,4}$ Faculty Mathematics and Science, Universitas Negeri Malang, Indonesia
}

\begin{abstract}
The aim of this study was to investigate reversible reasoning, particularly, mental action deployed by undergraduate students in problem situations of the inverse function. This case study compares the reversible reasoning of three high-achieving undergraduate students. These students were tested before with tests measuring their understanding of aspect function. Data collected through think aloud and clinical interviews depict strategies that the participants used to work back to find original function. the results show that three undergraduate students exhibit different reversible reasoning models when dealing with inverse function problems, consists of reversible rigid, analytic, and divergent strategy. Suggestion for further inquiry on improving knowledge and legitimation about reversible reasoning as valuable tools for teachers and students in classroom practice.
\end{abstract}

Keywords—reversibility; reversible reasoning; mental action; inverse function

\section{Introduction}

Reversibility is a mental process and conceptual understanding are two keys to developing current mathematical thinking and reasoning [1]-[5]. Some studies have acknowledged reversible reasoning and conceptual understanding independently such as addition, subtraction, multiplication, and division [1], [2], [4]-[8], but little has been known related to the relationship between concepts, especially those associated with the way students reverse their thoughts when dealing with fractions, exponents, and logarithms, trigonometric function, anti-derivative and derivative, function, and inverse. Despite its importance, the relationship between reversible reasoning and conceptual understanding lacks attention from researchers in mathematics education.

Rather than a structural perspective that occurs in negations and compensations [9], this investigation would refer to the reversible reasoning suggested by Krutetskii [10]. This mental process, which is experienced by students in reversing a problem, is known as reversible reasoning. Reversing a problem is not an easy task for students [11]-[15] because students tend to perceive a direct process and it's reversed as to two different things. Meanwhile, if the students are able to presume the two processes as equivalent and correlated, they can perform reversible reasoning which helps them develop stronger networking between concepts. In short, the students can think about the process from the beginning until the end or vice versa which as a result improve their ability to understand a mathematical problem.

The term reversible reasoning was formulated by Ramful [3], [16], [17] by adopting the definitions of reversible thinking suggested by [9], and [18] who stated that "students are able to anticipate and review a problem analytically (recall mentally)." Structurally, in reversing a problem students try to construct the source of the problem from its result. Reversible reasoning occurs based on a problem's operation, structure, and transformation [1], [3], [5], [14], [19]. This article is going to focus more on the structure of the problem which consists of the input (I), process $(\mathrm{P})$, and the result $(\mathrm{R})$ formulated in a notation developed by [5] $(\mathrm{I}(\mathrm{P}) \rightarrow \mathrm{R})$.

The structure of the problem is identical with a composition of functions $f(g(x)=h(x)$, where $g(x)$ refers to the input, $f(x)$ refers to the process, and $h(x)$ refers to the result. Reversible reasoning happens when the result and the process are used alternately [1] and when students possess an anticipation scheme in solving a problem (Hackenberg, 2010; Simon et al., 2016b; Simon et al, 2016b; Tzur \& Simon, 2004). Analysis of a composition of functions is limited to students' understanding [22]-[27]. Implicitly, some of the studies discussed reversible reasoning but not in detail. These studies had only involved translation (graph $\mathrm{f}(\mathrm{x}) \rightarrow \mathrm{f}(\mathrm{x}+\mathrm{k})$ or vice versa) to describe a function graph [25], [27], looked for a composition of functions $f(g(x))=h(x)$ [28], [29], or solved a composite $f(g(x))$ using the chain rule [30]-[32]. There has 
been no research reporting on the mental process experienced by students in reversing a structural relationship of a composition of functions which involves input, process, and result. This study would add variable changing (translation) to an unknown function, for example, $f(x)$ as the basis and then work on $\mathrm{f}(\mathrm{x}+\mathrm{k})$.

The results of a preliminary study suggested four types of reversible reasoning. It was reported that the students used the nature of a composition, an inverse function $(y=f(x))$, and mental construction by employing the properties of the similarity of functions through substitution or variable translation. This article would report on the results of research conducted on three undergraduate mathematical students with different types of reversible reasoning. We tried to elicit their responses and actions towards a composition of functions through an interview. This investigation attempted to reveal how the students reversed their mental process. As a result, we successfully identified what type of reversible reasoning they were using in solving a composition of functions. Findings of the current study would thus be expected to answer these questions: What reasoning did undergraduate mathematical students use in solving inverse functions problem situations? Is reversible or irreversible reasoning?

\section{Research Methods}

\subsection{Participants}

This study was conducted in one section of the course "research on school mathematics" at a state university in the east java during the even semester of 2017. We conducted a case study to three undergraduate mathematical students ( $\mathrm{S} 1$, S2, and S3) who were considered to have different reasoning types, especially in reversing a composition of functions. The subjects of the research were enrolled in the same course. We investigated their mental process as an individual and examined their reversible reasoning. The three students were selected based on their scores on a test on simple compositions. We asked for suggestions and recommendations from a mathematics professor in South Sulawesi regarding high-achieving students who were able to solve problems and communicate well (think aloud) in an interview. The participants were asked to uncover their thoughts. Initially, we put our main focus on S1 and S2; however, after examining the work result of S3, who used a variable changing strategy, we then decided to do further investigation on her mental process.

\subsection{Data Collection and Analysis}

The research team comprised of four members who assisted in several roles. During each interview, one of the researchers served as the interviewer and the other three acting as supervisors. All interviews are held in a study hall at the university during lunch periods. Interviews were held in
Indonesian, and responses were recorded and transcribed. Each interview was recorded with two cameras, one focused on the gestures and the interaction between researchers and students, and one focused on the student's written work. These videos were digitally combined into one file for analysis.

Clinical interviews were performed after analyzing the students' answers. The results of the analysis were then made as a reference to develop the interview guidelines. The purpose of the interview was to obtain a clear picture of how the students reflected on their answers. We focused on the students' mental process in reversing inverse functions problem and on how the students made use of information related to the problem. The interviews were conducted in a classroom and an auditorium. They were recorded using a camera: one camera was focused on the interaction between the researcher and the students while the other was used to observe the students' work. These videos were compiled in one file and analyzed.

The interviews followed the guidelines but were conducted informally in order that the students did not feel tense and intimidated. One or two tasks were assigned to the students. The students were required to think aloud while doing the tasks so that their mental processes could be described. They would think aloud their strategies to complete the tasks and voice their thoughts towards the tasks. The results of the clinical interviews were used to help us draw a conclusion on the students' mental process. Prior to the analysis of the interviews, we generated some assumptions related to types of reversible reasoning that might be developed by the students to solve a composition of functions. In this case, we referred to the mental process that could portray how concepts were constructed in the students' individual minds. The reversible reasoning type suggested was in line with the students' work. Some categories of the students' responses were reviewed to adjust to the research findings. After analyzing the students' work and the excerpts of the interview, we observed the clips to confirm and expand the findings and finally make an inference on the students' mental process.

\subsection{The Development of the Tasks}

Our purpose to develop the tasks was to analyze the students' mental process when they reversed a composition of functions, particularly the way the students associated their understanding with the inverse concept. We had developed [28] instrument by providing three types of problems of $H=$ $F \circ G$ type 1 (known $F$ and $G$, find $\mathrm{H}$ ), type 2 (known $F$ and $H$, find $\mathrm{G}$ ), and type 3 (known $H$ and $G$, find $\mathrm{F}$ ). Type 2 and type 3 were considered able to reveal the students' reversible reasoning. Some students admitted that the two types of problems (type 2 and type 3 ) were difficult to solve directly. The students tended to use a procedural strategy to obtain the result. For some students, however, this tendency would lead 
to their inability to drawing a conclusion. in addition, this type of problem is able to produce many mathematical ideas compared to type 3 . Therefore, in this article, we selected the Type 2 problem to investigate reversible reasoning. The three participants were asked to solve the following problem:

$$
\begin{gathered}
h(x):=\frac{x-5}{x+1} \text { and } g(x):=2 x-1, h \text { is the composite of } \\
h:=f \circ g, \text { what is } f(x+5) ?
\end{gathered}
$$

\section{Results and Discussion}

\subsection{Results}

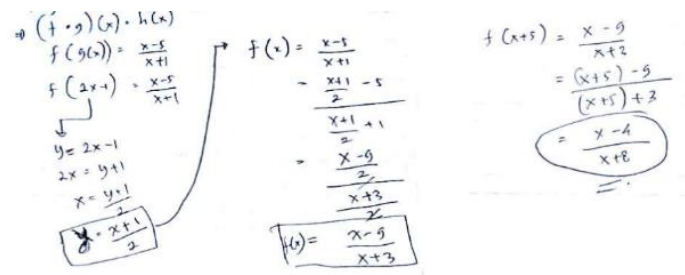

Figure 1. S1 answer to task

Type 2 problem $((f \circ g)=h(x))$ was aimed to find function $f(x+5)$ based on known $h(x)$ and $g(x)$. To solve the problem, $\mathrm{S} 1$ as the first participant used the properties of the composition $(f \circ g)=f(g(x))$, then she found the standard function $f(x)$, and made a connection between other known functions. S1 evaluated the structure of the problem by seeing $g(x)$ as the input, $f(x)$ as the process, and $h(x)$ as the result. S1 explicitly revealed that it was necessary to find the inverse function of $g(x)$

S1: Because $f(g(x))=h(x), h(x)$ and $g(x)$ are identified, then to find $f(x)$, function $g(x)$ must be inversed and $x$ on $h(x)$ should be replaced

S1 did not mention the identity properties of the composition which allows $g(x)$ to have an inverse function. As a result, she did not understand why the result of the inverse of $g(x)$ was substituted into function $h(x)$. According to the procedures, there was something missing from S1's mental activity: she did not mention the identity properties of the composition $f \circ g \circ \mathrm{g}^{-1}=h \circ g^{-1}$. S1 only understood the instrumental aspect of the properties. As was suggested by Skemp, she only focused on how to change $f(g(x))$ into $f(x)$ by making a connection between the initial concepts: $y=f(x)$ and $x=f^{-1}(x)$. S1 says:

S1: I found the inverse function of $g(x)=2 x+1$, to find $g^{-1}(x)$, I made an adjustment $y=2 x+1$, the result of the inverse of $x$ was $\frac{y-1}{2}$ or $g^{-1}(x)=\frac{x-1}{2}$

We observed how $\mathrm{S} 1$ found the inverse function of $y=2 x+1$. We found out that she was using a procedural strategy and reversing the operation verbally to justify the procedural strategy. She mentally reversed the order of the operation by seeing $2 x+1$. In line with [3], [16], [17], reversing an inverse of a function can be revealed diagrammatically based on the order of the operation.
The inverse of $g^{-1}(x)$ was substituted, replacing $x$ on $h(x)$ or $h\left(g^{-1}(x)\right)$ to find $f(x)$. Despite her lack of understanding of the properties of the composition, $\mathrm{S} 1$ could recognize how to find $f(x)$ from $f(g(x))=h(x)$. She realized that she needed to find the inverse function of $g^{-1}(x)$ and substituted it with $h(x)$. According to [10], basically, S1 had conducted an analytical investigation of the problem by observing the structure and reversing the operation verbally to validate her procedural strategy. In addition to that, S1 also mentioned that there was another strategy that could be used to solve a composition of functions, for example without having to change it to the standard function $f(x)$ ).

In spite of this, she still decided to choose to reverse the operation and do the substitution (Figure 1). It was assumed that S1's learning experience was the one that affected her decision. She might have learned how to solve a routine problem but did not try to understand every step of it, particularly that of the identity of a composition of functions.

Unlike S1, S2 drew a conclusion from observing the structural relationship between the input $g(x)$, the process $f(x)$, and the result $h(x)$. In solving the problem, S2 could understand the identity of a composition of functions. As a result, he attempted to find $(f(x))$ first and do substitution to find the function, but because $g(x)$ was linear $(2 x-1)$, he then decided to use another procedure to solve the problem. He stated that:

S2: $\quad$ because I need to find $f(x)$, both of the right sides of $f \circ g=h$ should be added with $g^{-1}$, so it can produce an identity $\cdots f=h \circ g^{-1}$. I did not do it that way because $\mathrm{g}(\mathrm{x})$ was linear.

$\mathrm{S} 2$ tried to reverse the problem by making an assumption from the structural relationship between functions in the composition. He separated fractions in $h(x)$ between the numerator and denominator, and vocalized (think aloud) how he changed $x-5$ and $x+1$ to make $2 x-1$ from variable $x$. $\mathrm{S} 2$ repeatedly made some assumptions using trial and error strategy.

S2 established a reciprocal relationship by changing $x-$ 5 and $x+1$ into $2 x-1$ and testing the solution whether it could produce $x-5$ and $x+1$. He did this because he realized that both sides of the function contained $2 x-1$; therefore he could find the standard function $(f(x))$.

Moreover, he performed a procedural process through substitution to find the desired function. S2's mental process in reversing the problem analytically consisted of identifying the structure of the problem, making assumptions using trial and error strategy, building a reciprocal relationship, seeing the similarity of both sides of the structure, and drawing a conclusion on the standard function. This mental process shown by S2 is corroborated with Hackenberg's opinions [21], [33], [34] which state that it is necessary to establish a reciprocal relationship in solving a problem. S2's mental process is also confirmed by [35] who argues that identifying the structure of the problem and drawing a conclusion is 
equally important in problem-solving. We perceived that S2 was able to reverse the composition of functions analytically with a good conceptual understanding of identity. In addition to that, we found out that $\mathrm{S} 2$ could see the problem from a different perspective.

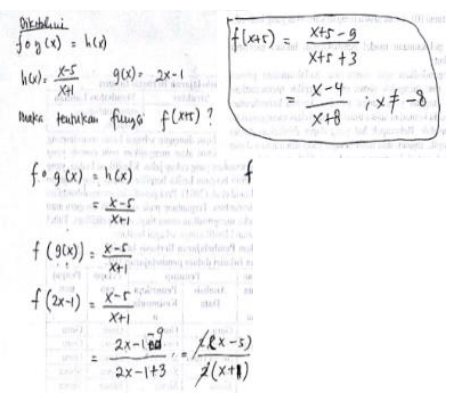

Figure 2. S3 answer to task

The other participant, S3, was also able to make a structural relationship between the input, the process, and the result. In the beginning, she made use of the process similar to that performed by S1 and S2, but then she tried to simplify it by reversing the problem without finding the standard function $(f(x))$. She attempted to connect translation of function $\mathrm{f}(2 \mathrm{x}-1)$ to $\mathrm{f}(\mathrm{x}+5)$. She answered:

S3: $\quad$ I understand that to solve this problem I need to find the inverse function or use another way, probably I can simplify it without having to find $f(x)$

S3 constructed her assumptions by utilizing function translation and she was thinking of changing $x$ in $2 x-1$ to generate $x+5$ without having to change $h(x)$. S3 investigated $x$ in $2 x-1$. She felt that it had to be divided by 2 and the constant must also be divided by 2 and subtracted by 1 to get positive 5 . She then compared the results by identifying the properties of the composition $\left(g \circ g^{-1}\right)$ and using a trial and error strategy such as the one used by $\mathrm{S} 2$. She was later aware of her mistake: she assumed $x$ in $2 x-1$ is $\frac{x-4}{2}$, but it did not generate $x+5$.

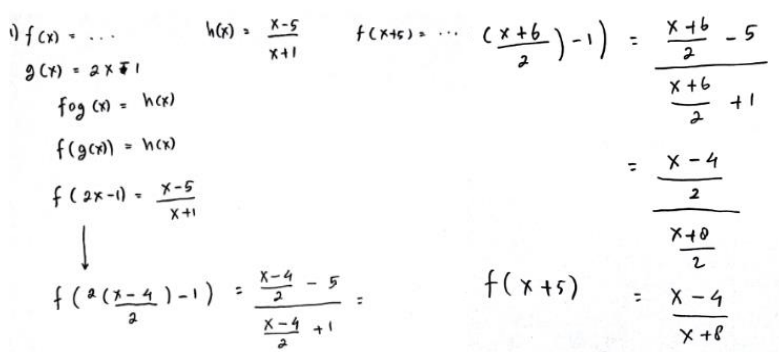

Figure 3. S3 answer to task

S3 built a translation relationship between functions without having to find the standard function $(f(x))$. Analytically, her mental process showed that S3 used a conceptual understanding of the composition identity, reciprocal relationship, the similarity of the function, also function translation to simplify the process. This finding is quite intriguing because S3 could perform the three schemes of solving a composition of function at the same time. She was able to establish a relationship between the schemes and use them together to solve the problem. Compared to S2 who was only able to recognize these, S3 was able to make use of the conceptual understanding scheme, see the problem from a new perspective, and develop the scheme to solve the problem [36]-[39] (Figure 3). The difference between these three students lied on their strategies and mental process in reversing a problem. In conclusion, S1, S2, and S3 had different types of reversible reasoning.

We involved one of mathematical giftedness called the analytic type (verbal-logic) [10] and combined it with the development schemes (at the intra, inter, and trans level) [39]. The analytic type refers to the mental action to reverse a problem by using the properties of the composition $\left(f(x)=h\left(g^{-1}(x)\right)\right.$, see the composition structure and to make an adjustment to find the inverse $(y=g(x))$, and involve the nature of the similarity of the function through substitution or variable translation. The property scheme was related to two important aspects: understanding the composition of functions and coordinating conditions such as the information about the structure of the composition and known functions.

Rigid reversible type refers to the mental action to reverse a problem conceptually by using the properties of the composition and an adjustment to find the inverse. At this level, students could only make use of the properties of the composition because they are not able to reveal other strategies even though they do acknowledge the presence of the strategies. Analytic reversible type refers to the mental action to reverse a problem by examining the structural relationship of the composition without employing conceptual strategies (the properties of the composition and adjustment). Students correlate the source and the result on the composition of functions to obtain the standard form of the function. At this level, undergraduate mathematical students may be informed about the procedural strategy that is by using an inverse, but they do not use it to solve a problem. Divergent strategy reversible type refers to the mental action to reverse a problem by not only using the conceptual strategy but also coordinating the structural relationship of functions without changing them into their standard form $(f(x))$ or transforming the variables (mental processing).

\subsection{Discussion}

This investigation could successfully describe each participant's type of reversible reasoning in solving an inverse function situation. It also revealed how the students tried to understand the problem given and how they reversed it. The results of the analysis proved that the mental process could indicate the difference in schemes that each undergraduate mathematical student possesses. The results also suggested that the difference in schemes should be 
perceived as the mental action of an individual or an undergraduate mathematical student in this case to build a relationship between their conceptual understanding and other perspectives in reversing a problem. These findings are in line with other research findings [36]-[40].

We combined Krutetskii's analytical thinking and the development stages of [39] by formulating types of students' reversible reasoning theoretically. However, this should be reviewed further because one of the subjects of this research, S1, had an instrumental understanding of identity properties and mental operation order [3], [5], [16], [17], [19], [21], [33], [34]. We, thus, suggest the use of rigid reversible due to S1's inability to understand the identity concept. Unlike S1, S2 can be categorized into an Analytic reversible type because he could understand the existence of the identity concept although he did not use it in his work. In addition, S2 used another point of view in reversing the problem such as shown in Table 1. He was able to establish a reciprocal relationship between functions in the composition and identify the similarity of the structure of the functions.

The other student, S3, belonged to the divergent strategy reversible type because she could reveal the identity concept, establish a reciprocal relationship between functions in the composition and identify the similarity of the structure of the functions (such as what S2 did). She used another perspective without having to find the standard function $(\mathrm{f}(\mathrm{x}))$, involved function translation, and at the same time performed a mental operation in reversing a problem. Findings, thus, indicated two things: First, related to the concept of a composition of functions: the three undergraduate mathematical students were able to recognize the use of identity concept in solving a problem; Second, the students were able to develop their thoughts in reversing a problem based on other perspectives; they constructed their background knowledge to find another strategy to solve an inverse function situation without making the identity properties as the initial knowledge.

We assume that the difference in ways of the students reversed a problem in a composition could reflect their difference in how their reflective abstraction operated [37] as well as how they experience learning in the classroom. We believe that undergraduate mathematical students' mental processes in different schemes of knowledge can be combined through a learning interaction so that the students can understand the concept of inverse functions. Despite its lack of contribution to the learning process, this research can be useful to enrich learning instruction.

Based on the characteristics and mental actions performed (Ithriah, S3, and S2), types of reversible reasoning could be summarized in the following table.

\begin{tabular}{ll}
\hline Types & Indications \\
\hline Reversible & Having a simple understanding of the \\
Rigid & identity concept \\
& Mental Action: \\
\hline
\end{tabular}

"to find $f(x)$, function $g(x)$ must be inversed and $x$ on $h(x)$ should be replaced"

Make an adjustment to find the inverse

Mental Action:

to find $g^{-1}(x), g(x)$ must be adjusted with

Find function by procedures

Mental Action:

Find $f(x+5)$ through $x+5$ in $f(x)$

Know another strategy, but cannot reveal it Mental Action:

"there was another way to find the function, but I am already used to this strategy"

Reversible Conscious about the properties of the Analytic composition and inverse Mental Action:

"I was looking for ways to keep both sides the same"

Make an assumption from the structural relationship between functions in a compositions

Mental Action:

"How to apply $f((2 x+1)$ so that the right side also contains $2 x+1$ "

Draw an inference on the standard form through the similarity of the structures Mental Action:

"because $f(2 x+1)=\frac{(2 x+1)+1}{2}$ both sides are similar, then $f(x)=\frac{x+1}{2}$,

Find function through substitution

Mental Action

"right-side and left-side equally is $f(2 x+1)$, so it is could be concluded its $f(x)$ "

Reversible Possess several ways to find original Divergent condition

Mental Action:

"This problem can be solved in various ways; we can use inverse or substitution" Make an assumption from function translation

Mental Action:

"I think about how to change $f(2 x+1)$ into $f(x+5)$ "

Involve a mental process to draw a conclusion

Mental Action:

"because $2 x+1$, I divided it by a half, added a half, thus I got 5 "

Do the substitution based on the result of the function translation

Mental Action

" $f(2 x+1) \rightarrow f(x+1)$ can be written

$\left(2\left(\frac{x+4}{2}\right)+1\right)$ equals $f(x+5) "$

\section{Conclusion}

This study pursued to apprehend those precise instances where undergraduate students gave evidence of reversing 
their thinking. To portrait those moments, I provide the three participants with a set of progressive tasks, where the complexity of the situation was increased by varying the type of strategy used in the inverse function. The main conclusions of this study are outlined below.

Firstly, this study shows that the problem situation promotes the participants to solve it with reversible reasoning, from original situations $(\mathrm{f}(\mathrm{g}(\mathrm{x})))$ to engender result situation $(\mathrm{h}(\mathrm{x}))$. Secondly, three types of reversible reasoning were found from participants' mental activities, namely rigid, analytic, and divergent strategies. Rigid reversible inclined reversing the order of functions by exchanging $\mathrm{x}$ and $\mathrm{y}$ and solving for $y$. Reversible analytic disposed to reversing the process from function to inverse, reversing the situation, and considering similarities between two sides of the situation with the algebra approach. Reversible divergent tend to view the problem situation in other representations or strategies, and developed the process back and forth to ensure the complete process is carried out compatible.

For future research, To date, the mathematics education literature has provided little theoretical guidance on promoting reversible reasoning. By building on the construction to find the inverse function process, we have developed three types of reversible reasoning for this purpose. Our work has focused on developing reversible reasoning by using the original concept to build the reverse, there are other ways to think about that. Another claims that reversible reasoning is essential to achieve a deep understanding that enables advanced mathematical thinking. We argue that reversible reasoning to become the valuable tool for understanding that it could be students must be guided to explicitly practice characteristics of reversible reasoning, teachers' knowledge about reversible reasoning should be improved, and reversible reasoning must undergo a process of legitimation. Researchers should investigate the existence a link between teaching that attends to linking multiple representations and the development of reversible reasoning in classroom, making teacher and students aware of the utility of reversible reasoning and its different roles in mathematics education, and last and most important, teachers and students are not trained to use reversible reasoning to solve tasks in their classes.

Reversible reasoning is also involved in other knowledge like calculus and one observation that students were often constrained in deriving the graph of a function starting from the graph of derivative of function and students could not readily construct the relationship between the derivative and anti-derivative graph. Finally, further study needs to examine situational and condition that cause students making reversible reasoning and its steps.

\section{Acknowledgement}

This work was supported by Lembaga Pengelola Dana Pendidikan (LPDP) Indonesia under grant no. 20161141081895

\section{References}

[1] Steffe L P and Olive J, Children's fractional knowledge, Springer Science \& Business Media, 2009.

[2] Flanders S T, Investigating Flexibility, Reversibility, and Multiple Representation in a Calculus Environment, University of Pittsburg, 2014.

[3] Ramful A, "Reversible reasoning in fractional situations: Theorems-in-action and constraints," J. Math. Behav., vol. 33, pp. 119-130, 2014.

[4] Lee M Y and Hackenberg A J., "Relationships between fractional knowledge and algebraic reasoning: The case of Willa, Int. J. Sci. Math. Educ., vol. 12, no. 4, pp. 975-1000, 2014.

[5] Simon M A Kara M Placa N and Sandir H, "Categorizing and promoting reversibility of mathematical concepts," Educ. Stud. Math. vol. 93, no. 2, pp. 137-153, 2016.

[6] Adi H., "Development and Intellectual of Thought in Equation," J. Res. Math. Educ. vol. 9, no. 3, pp. 204-213, 1978.

[7] Dougherty B J Bryant D P Bryant B R Darrough R L and Pfannenstiel K H., "Developing concepts and generalizations to build algebraic thinking: The reversibility, flexibility, and generalization approach," Interv. Sch. Clin., vol. 50, no. 5, pp. 273-281, 2015.

[8] Tunç-Pekkan Z., "An analysis of elementary school children's fractional knowledge depicted with a circle, rectangle, and number line representations," Educ. Stud. Math., vol. 89, no. 3, pp. 419-441, 2015.

[9] Inhelder and Piaget, The Growth of Logical Thinking From Child to Adolescence, Basic Books, Inc, New York, 1958.

[10] Krutetskii V A., "The psychology of mathematical abilities", Schoolchildren, vol. 8, no. 3, 1976.

[11] Gray E M and Tall D O., "Duality, ambiguity, and flexibility: A "perceptual" view of simple arithmetic, J. Res. Math. Educ., vol. 25, no. 2, pp. 116, 1994.

[12] Selter C Prediger S Nührenbörger $M$ and Hußmann S, "Taking away and determining the difference-a longitudinal perspective on two models of subtraction and the inverse relation to addition," Educ. Stud. Math., vol. 79, no. 3, pp. 389-408, 2012.

[13] Anderson Norton and Jesse L. M. Wilkins, "The splitting group," J. Res. Math. Educ., vol. 43, no. 5, pp. 557, 2012.

[14] Hackenberg A J and Lee M Y., "Relationships between students' fractional knowledge and equation writing," $J$. Res. Math. Educ., vol. 46, no. 2, pp. 196-243, 2015.

[15] Sangwin C J and Jones I, "Asymmetry in student achievement on multiple-choice and constructed-response items in reversible mathematics processes," Educ. Stud. Math., vol. 94, no.2, pp. 205-222, 2017.

[16] Ramful A, Reversible Reasoning In Multiplicative Situations: Conceptual Analysis, Affordances, And Constra1nts Dissertation, 2009.

[17] Ramful A, "Reversible reasoning and the working backward problem-solving strategy," Aust. Math. Teach., vol. 71, no. 4, pp. 28-32, 2015.

[18] Wong B, "The Relationship between Piaget's Concept of Reversibility and Arithmetic Performance among Second Grades," Annu. Meeting Am. Educ. Res. Assoc., 1977.

[19] Simon M A Placa N and Avitzur A, "Participatory and anticipatory stages of mathematical concept learning: Further empirical and theoretical development," J. Res. Math. Educ., vol. 47, no. 1, pp. 63-93, 2016.

[20] Tzur R and Simon M., "Distinguishing two stages of mathematics conceptual learning," Int. J. Sci. Math. Educ., vol. 2, no. 2, pp. 287-304, 2004.

[21] Hackenberg A J, "Students' reasoning with reversible multiplicative relationships," Cogn. Instr., vol. 28, no. 4, pp. 383-432, 2010.

[22] Bagley S Rasmussen C and Zandieh M, "Inverse, composition, and identity: The case of function and linear transformation," J. Math. Behav. vol. 37, pp. 36-47, 2015.

[23] Dubinsky E and Wilson R T, "High school students' understanding of the function concept," J. Math. Behav., vol. 32, no. 1, pp. 83-101, 2013.

[24] Leron U and Paz T, "Functions via everyday actions: Support or obstacle?" J. Math. Behav. vol. 36, pp. 126-134, 2014. 
[25] Proulx J, "Mental mathematics with mathematical objects other than numbers: The case of operation on functions," $J$. Math. Behav., vol. 39, pp. 156-176, 2015.

[26] Tabach $M$ and Nachlieli T, "Classroom engagement towards using definitions for developing mathematical objects: the case of function," Educ. Stud. Math., vol. 90, no. 2, pp. 163187, 2015.

[27] Weber E and Thompson P W, "Students' images of twovariable functions and their graphs," Educ. Stud. Math., vol. 87, no. 1, pp. 67-85, 2014.

[28] Arnon I Cottrill J and Dubinsky E, APOS Theory Springer Science Business Media New York, 2014.

[29] Bowling S, Conceptions of function composition in college precalculus students, May, 2014.

[30] Jojo Z M M Maharaj A and Brijlall D, "From human activity to conceptual understanding of the chain rule," J. Res. in Mathematics Educ., vol. 2, no. 1, pp. 77-99, 2013.

[31] Kontorovich I, "Students' confusions with reciprocal and inverse functions," Int. J. Math. Educ. Sci. Technol., vol. 48, no. 2, pp. 278-284, 2017.

[32] Park J, "Is the derivative a function? If so, how do we teach it?" Educ. Stud. Math., vol. 89, no. 2, pp. 233-250, 2015.

[33] Hackenberg A J, 2005 Construction of algebraic reasoning and mathematical caring relations October 2003.

[34] Hackenberg A J, "The fractional knowledge and algebraic reasoning of students with the first multiplicative concept," J. Math. Behav., vol. 32, no. 3, pp. 538-563, 2013.

[35] Ian Jones and Dave Pratt, "A substituting meaning for the equals sign in arithmetic notating tasks," J. Res. Math. Educ., vol. 43, no. 1, 2012.

[36] Haciomeroglu E S Aspinwall L and Presmeg N C, "Contrasting cases of calculus students' understanding of derivative graphs," Math. Think. Learn., vol. 12, no. 2, pp. 152-176, 2010.

[37] García M Llinares $S$ and Sánchez-Matamoros G, "Characterizing thematized derivative schema by the underlying emergent structures," Int. J. Sci. Math. Educ., vol. 9, no. 5, pp. 1023-1045, 2011.

[38] Hong Y Y and Thomas M O J, "Graphical construction of a local perspective on differentiation and integration," Math. Educ. Res. J., vol. 27, no. 2, pp. 183-200, 2015.

[39] Baker B Cooley L and Trigueros M, "A calculus graphing schema," J. Res. Math. Educ., vol. 18, no. 3, pp. 163-179, 2000.

[40] Asiala M Cottrill J Dubinsky E and Schwingendorf K E, "The development of students' graphical understanding of the derivative," J. Math. Behav., vol. 16, no. 4, pp. 399-431, 1997. 